\title{
Holder's Inequality for the Polar Derivative of Polynomial Having All Zeros inside a Disk
}

\author{
R. Lal ${ }^{*}$ \\ Department of Mathematics, V.S.K.C. Govt. PG College, Dakpathar, Dehradun, Uttrakhand, India
}

Received 4 August 2019, accepted in final revised form 22 September 2019

\begin{abstract}
In this paper, we have proved an $\mathrm{L}^{\mathrm{p}}$ inequality for polar derivative of the polynomial having all its zeros in the disk $|z| \leq k, k \leq 1$. Our result generalizes and improves the earlier known results.
\end{abstract}

Keywords: Polynomials; $\mathrm{L}^{\mathrm{r}}$ inequality; Zeros; Polar derivative of polynomials.

(c) 2020 JSR Publications. ISSN: 2070-0237 (Print); 2070-0245 (Online). All rights reserved. doi: http://dx.doi.org/10.3329/jsr.v12i1.42476

J. Sci. Res. 12 (1), 75-81 (2020)

\section{Introduction}

Let $p(z)=\sum_{j=0}^{n} a_{j} z^{j}$ be a polynomial of degree $\mathrm{n}$ and $p^{\prime}(z)$ be its derivative, then for $r>0$,

$$
\left\{\int_{0}^{2 \pi}\left|p^{\prime}\left(e^{i \theta}\right)\right|^{r} d \theta\right\}^{\frac{1}{r}} \leq n\left\{\int_{0}^{2 \pi}\left|p\left(e^{i \theta}\right)\right|^{r} d \theta\right\}^{\frac{1}{r}}
$$

Inequality (1.1) is sharp and equality holds for polynomial $p(z)=\alpha z^{n}, \alpha \neq 0$.

Inequality (1.1) for $r \geq 1$ is due to Zygmund [1], who proved it for all trigonometric polynomials of degree $n$ and not only for those which are of the form $p\left(e^{i \theta}\right)$. Arestov [2] proved that (1.1) remains true for $0<r<1$ as well. Lal [3] also has generalized inequality (1.1).

If we let $r \rightarrow \infty$ in (1.1) and make use of well-known fact from analysis [4] that

$$
\lim _{r \rightarrow \infty}\left\{\frac{1}{2 \pi} \int_{0}^{2 \pi}\left|p\left(e^{i \theta}\right)\right|^{r} d \theta\right\}^{\frac{1}{r}}=\max _{|z|=1}|p(z)|,
$$

we get the following inequality

$$
\max _{|z|=1}\left|p^{\prime}(z)\right| \leq n \max _{|z|=1}|p(z)|
$$

Inequality (1.3) is a classical result due to Bernstein [5].

${ }^{*}$ Corresponding author: rlkeshtwal@gmail.com 
If we restrict ourselves to the class of polynomials having no zeros in $|z|<1$, then inequality (1.1) can be sharpened. In fact, in this case the following results are also known.

Theorem A. If $p(z)$ is a polynomial of degree $n$ having no zero in $|z|<1$, then for each $r>0$,

$$
\left\{\int_{0}^{2 \pi}\left|p^{\prime}\left(e^{i \theta}\right)\right|^{r} d \theta\right\}^{\frac{1}{r}} \leq n C_{r}\left\{\int_{0}^{2 \pi}\left|p\left(e^{i \theta}\right)\right|^{r} d \theta\right\}^{\frac{1}{r}},
$$

where

$$
C_{r}=\left\{\frac{1}{2 \pi} \int_{0}^{2 \pi}\left|1+e^{i \alpha}\right|^{r} d \alpha\right\}^{-\frac{1}{r}} .
$$

In above inequality, equality occurs for $p(z)=\alpha z^{n}+\beta,|\alpha|=|\beta|$.

For $r \geq 1$, Theorem A was proved by de-Bruijn [6] and later independently proved by Rahman [7].

Aziz and Rather [8] proved the following result for the polar derivative of a polynomial $p(z)$.

Theorem B. If $p(z)$ is a polynomial of degree $n$ having all its zeros in $|z| \leq k, k \leq 1$, then for every real or complex number $\alpha$ with $|\alpha| \geq k$,

$$
\max _{|z|=1}\left|D_{\alpha} p(z)\right| \geq n\left(\frac{|\alpha|-k}{1+k}\right) \max _{|z|=1}|p(z)| \text {. }
$$

Inequality (1.5) is best possible and equality occurs for $p(z)=(z-k)^{n}$ with real $\alpha \geq k$.

Recently integral inequalities have been extended to polar derivatives. In this direction, the following result was obtained by Govil et. al. [9].

Theorem C. If $p(z)$ is a polynomial of degree $n$ having no zero in $|z|<1$, then for $r \geq 1$ and for every real or complex number $\alpha$ with $|\alpha| \geq 1$,

$$
\left(\int_{0}^{2 \pi}\left|D_{\alpha} p\left(e^{i \theta}\right)\right|^{r} d \theta\right)^{\frac{1}{r}} \leq n(|\alpha|+1) F_{r}\left(\int_{0}^{2 \pi}\left|p\left(e^{i \theta}\right)\right|^{r} d \theta\right)^{\frac{1}{r}},
$$

where

$$
F_{r}=\left(\frac{2 \pi}{\int_{0}^{2 \pi}\left|1+e^{i \theta}\right|^{r} d \theta}\right)^{\frac{1}{r}} .
$$

In the limiting case, when $r \rightarrow \infty$, the above inequality is sharp and equality holds for the polynomial $p(z)=\lambda+\mu z^{n},|\lambda|=|\mu|$.

Dewan et. al. [10] obtained a generalization of Theorem B in the sense that maximum of $|p(z)|$ on $|z|=1$ on the right hand side of (1.5) is replaced by a factor involving the integral mean of $p(z)$ on $|z|=1$. In fact, they proved

Theorem D. If $p(z)$ is a polynomial of degree $n$ having all its zeros in $|z| \leq k, k \leq 1$, then for every real or complex number $\alpha$ with $|\alpha| \geq k$, and for each $r>0$ 


$$
n(|\alpha|-k)\left(\int_{0}^{2 \pi}\left|p\left(e^{i \theta}\right)\right|^{r} d \theta\right)^{\frac{1}{r}} \leq\left(\int_{0}^{2 \pi}\left|1+k e^{i \theta}\right|^{r} d \theta\right)^{\frac{1}{r}} \max _{|z|=1}\left|D_{\alpha} p(z)\right|
$$

The result is best possible for sufficiently large $\mathrm{r}$ and equality holds for $p(z)=(z-k)^{n}$ with real $\alpha \geq k$.

Aziz and Shah [11], for the class of polynomials $p(z)=a_{n} z^{n}+\sum_{j=\mu}^{n} a_{n-j} z^{n-j}, 1 \leq \mu \leq n$, having all zeros in $|z| \leq k, k \leq 1$ proved the following

Theorem E. If $p(z)=a_{n} z^{n}+\sum_{i=1}^{n} a_{n-j} z^{n-j}, 1 \leq \mu \leq n$, is a polynomial of degree $n$ having all its zeros in $|z| \leq k, k \leq 1$, then for each $r>0$,

$$
n\left\{\int_{0}^{2 \pi}\left|p\left(e^{i \theta}\right)\right|^{r} d \theta\right\}^{\frac{1}{r}} \leq\left\{\int_{0}^{2 \pi}\left|1+k^{\mu} e^{i \theta}\right|^{r} d \theta\right\}^{\frac{1}{r}} \max _{|z|=1}\left|p^{\prime}(z)\right| .
$$

Now, if we involve the coefficients of a polynomial, then we obtain a result which gives an improvement as well as a generalization of Theorem D and in a particular case Theorem E. More precisely, we prove

Theorem 1. If $p(z)=a_{n} z^{n}+\sum_{j=\mu}^{n} a_{n-j} z^{n-j}, 1 \leq \mu \leq n$, is a polynomial of degree $n$ having all its zeros in $|z| \leq k, k \leq 1$, then for every real or complex number $\alpha$ with $|\alpha| \geq A_{k, \mu}$, and for each $r>0, p>1, q>1$ with $\frac{1}{p}+\frac{1}{q}=1$, we have

$$
\begin{aligned}
n\left(|\alpha|-A_{k, \mu}\right)\left(\int_{0}^{2 \pi}\left|p\left(e^{i \theta}\right)\right|^{r} d \theta\right)^{\frac{1}{r}} \\
\leq\left(\int_{0}^{2 \pi}\left|1+A_{k, \mu} e^{i \theta}\right|^{r p} d \theta\right)^{\frac{1}{r p}}\left(\int_{0}^{2 \pi}\left|D_{\alpha} P\left(e^{i \theta}\right)\right|^{r q} d \theta\right)^{\frac{1}{r q}},
\end{aligned}
$$

where

$$
A_{k, \mu}=\frac{n\left|a_{n}\right| k^{2 \mu}+\mu\left|a_{n-\mu}\right| k^{\mu-1}}{n\left|a_{n}\right| k^{\mu-1}+\mu\left|a_{n-\mu}\right|} .
$$

The following result is an immediate consequence by letting $q \rightarrow \infty$ (so that $p \rightarrow 1$ ) in Theorem 1.

Corollary 2. If $p(z)=a_{n} z^{n}+\sum_{j=\mu}^{n} a_{n-j} z^{n-j}, 1 \leq \mu \leq n$, is a polynomial of degree $n$ having all its zeros in $|z| \leq k, k \leq 1$, then for every real or complex number $\alpha$ with $|\alpha| \geq A_{k, \mu}$ and for each $r>0$

$$
n\left(|\alpha|-A_{k, \mu}\right)\left(\int_{0}^{2 \pi}\left|p\left(e^{i \theta}\right)\right|^{r} d \theta\right)^{\frac{1}{r}} \leq\left(\int_{0}^{2 \pi}\left|1+A_{k, \mu} e^{i \theta}\right|^{r} d \theta\right)^{\frac{1}{r}} \max _{|z|=1}\left|D_{\alpha} p\left(e^{i \theta}\right)\right|
$$

where $A_{k, \mu}$ is as defined in Theorem 1 . 
Remark 3. To prove Theorem 1 as an improvement as well as a generalization of Theorem $\mathrm{D}$, it is sufficient to show that

$$
A_{k, 1} \leq k,
$$

that is

$$
\frac{n\left|a_{n}\right| k^{2}+\left|a_{n-1}\right|}{n\left|a_{n}\right|+\left|a_{n-1}\right|} \leq k,
$$

which is equivalent to

$$
n\left|a_{n}\right| k^{2}+\left|a_{n-1}\right| \leq n\left|a_{n}\right| k+\left|a_{n-1}\right| k,
$$

that is

$$
n\left|a_{n}\right| k(k-1) \leq\left|a_{n-1}\right|(k-1)
$$

implies

$$
n\left|a_{n}\right| k \geq\left|a_{n-1}\right|, \quad \text { (since } k \leq 1 \text { ) }
$$

which is true due to Lemma 2.2 for $\mu=1$. Hence, we show that Theorem 1 is an improvement as well as an generalization of Theorem D.

Dividing both sides of (1.10) by $|\alpha|$ and letting $|\alpha| \rightarrow \infty$, we get the following result.

Corollary 4. If $p(z)=a_{n} z^{n}+\sum_{j=\mu}^{n} a_{n-j} z^{n-j}, 1 \leq \mu \leq n$, is a polynomial of degree $\mathrm{n}$ having all its zeros in $|z| \leq k, k \leq 1$, then for each $r>0$

$$
n\left(\int_{0}^{2 \pi}\left|p\left(e^{i \theta}\right)\right|^{r} d \theta\right)^{\frac{1}{r}} \leq\left(\int_{0}^{2 \pi}\left|1+A_{k, \mu} e^{i \theta}\right|^{r} d \theta\right)^{\frac{1}{r}} \max _{|z|=1}\left|p^{\prime}(z)\right|,
$$

where $A_{k, \mu}$ is as defined in Theorem 1.

Remark 5. The above inequality gives better bound than the bound obtained from inequality (1.8) of Theorem E. To prove this, it is sufficient to show that $A_{k, \mu} \leq k^{\mu}$. As we have proved earlier that $A_{k, 1} \leq k$, on similar lines, $A_{k, \mu} \leq k^{\mu}$ can be proved easily by using Lemma 2.2.

\section{Lemmas}

For the proofs of the above stated theorems, we need the following lemmas.

Lemma 2.1. If $p(z)=a_{n} z^{n}+\sum_{j=\mu}^{n} a_{n-j} z^{n-j}, 1 \leq \mu \leq n$, is a polynomial of degree $\mathrm{n}$ having all its zeros in $|z| \leq k, k \leq 1$, and let $q(z)=z^{n} p \overline{\left(\frac{1}{\bar{z}}\right)}$, then

$$
k^{\mu}\left|p^{\prime}(z)\right| \geq\left|q^{\prime}(z)\right| \text { for }|z|=1,
$$

The above lemma is due to Aziz and Shah [11, Lemma 2].

Lemma 2.2. If $p(z)=a_{n} z^{n}+\sum_{j=\mu}^{n} a_{n-j} z^{n-j}, 1 \leq \mu \leq n$, is a polynomial of degree $n$ having all its zeros in $|z| \leq k, k \leq 1$ and $q(z)=z^{n} \overline{p\left(\frac{1}{\bar{z}}\right)}$, then 


$$
\left|q^{\prime}(z)\right| \leq A_{k, \mu}\left|p^{\prime}(z)\right| \text { for }|z|=1,
$$

where $A_{k, \mu}$ is as defined in Theorem 1 ,

and

$$
\frac{n}{\mu}\left|\frac{a_{n}}{a_{n-\mu}}\right| \geq\left(\frac{1}{k^{\mu}}\right) .
$$

The above lemma is proved by others $[7,12]$.

\section{Proof of the theorem}

Proof of Theorem 1. Since $p(z)$ has all its zeros in $|z| \leq k, k \leq 1$, therefore by Lemma 2.2, we have for $|z|=1$

$$
\left|q^{\prime}(z)\right| \leq A_{k, \mu}\left|p^{\prime}(z)\right| \text {. }
$$

Since $q(z)=z^{n} p\left(\frac{1}{\bar{z}}\right)$, then we have $p(z)=z^{n} q\left(\frac{1}{\bar{z}}\right)$. This gives

$$
z p^{\prime}(z)=n z^{n} \overline{\left(\frac{1}{\bar{z}}\right)}-z^{n-1} q^{\prime}\left(\frac{1}{\bar{z}}\right)
$$

If $z=e^{i \theta}, 0 \leq \theta<2 \pi$, then from (3.2), we get

$$
\begin{aligned}
& \left|p^{\prime}\left(e^{i \theta}\right)\right|=\left|e^{i \theta} p^{\prime}\left(e^{i \theta}\right)\right| \\
& =\mid n e^{i n \theta} \overline{q\left(e^{i \theta}\right)}-e^{i(n-1) \theta} \overline{q^{\prime}\left(e^{i \theta}\right) \mid} \\
& =\left|n e^{-i n \theta} q\left(e^{i \theta}\right)-e^{-i(n-1) \theta} q^{\prime}\left(e^{i \theta}\right)\right| \\
& =\left|n q\left(e^{i \theta}\right)-e^{i \theta} q^{\prime}\left(e^{i \theta}\right)\right| .
\end{aligned}
$$

Equivalently,

$$
\left|p^{\prime}(z)\right|=\left|n q(z)-z q^{\prime}(z)\right| \text { for }|z|=1 \text {. }
$$

Using (3.3) in inequality (3.1), we get for $|z|=1$

$$
\left|q^{\prime}(z)\right| \leq A_{k, \mu}\left|n q(z)-z q^{\prime}(z)\right|
$$

Now for every real or complex number $\alpha$ with $|\alpha| \geq A_{k, \mu}$, we have for $|z|=1$

$$
\begin{aligned}
& \left|D_{\alpha} p(z)\right|=\left|n p(z)+(\alpha-z) p^{\prime}(z)\right| \\
& \geq|\alpha|\left|p^{\prime}(z)\right|-\left|n p(z)-z p^{\prime}(z)\right|,
\end{aligned}
$$

which gives by interchanging the roles of $p(z)$ and $q(\mathrm{z})$ in (3.3) for $|z|=1$ that

$$
\begin{aligned}
& \left|D_{\alpha} p(z)\right| \geq|\alpha|\left|p^{\prime}(z)\right|-\left|q^{\prime}(z)\right| \\
& \geq|\alpha|\left|p^{\prime}(z)\right|-A_{k, \mu}\left|p^{\prime}(z)\right| \quad \text { (using (3.4) } \\
& =\left(|\alpha|-A_{k, \mu}\right)\left|p^{\prime}(z)\right| .
\end{aligned}
$$

Again, since $p(z)$ has all its zeros in $|z| \leq k, k \leq 1$, therefore by Gauss-Lucas theorem $p^{\prime}(z)$ has all its zeros in $|z| \leq k, k \leq 1$ and thus the polynomial 


$$
z^{n-1} \overline{p^{\prime}\left(\frac{1}{\bar{z}}\right)}=n q(z)-z q^{\prime}(z)
$$

has all its zeros in $|z| \geq \frac{1}{k}, \frac{1}{k} \geq 1$. Therefore, it follows from (3.4) that the function

$$
w(z)=\frac{z q^{\prime}(z)}{A_{k, \mu}\left(n q(z)-z q^{\prime}(z)\right)}
$$

is analytic for $|z| \leq 1$ and $|w(z)| \leq 1$ for $|z| \leq 1$. Furthermore, $w(0)=0$ and so the function $1+A_{k, \mu} w(z)$ is subordinate to the function $1+A_{k, \mu} z$ for $|z| \leq 1$. Hence, by a well-known property of subordination [13], we have for $r>0$ and for $0 \leq \theta<2 \pi$,

$$
\int_{0}^{2 \pi}\left|1+A_{k, \mu} w\left(e^{i \theta}\right)\right|^{r} d \theta \leq \int_{0}^{2 \pi}\left|1+A_{k, \mu} e^{i \theta}\right|^{r} d \theta
$$

Now,

$$
1+A_{k, \mu} w(z)=\frac{n q(z)}{n q(z)-z q^{\prime}(z)},
$$

Which, with the help of (3.3), for $|z|=1$ gives

$$
\begin{aligned}
& \left|1+A_{k, \mu} w(z)\right|=\frac{n|q(z)|}{\left|p^{\prime}(z)\right|} \\
& =\frac{n|p(z)|}{\left|p^{\prime}(z)\right|} .
\end{aligned}
$$

Using (3.5) in the above inequality, we get for $|z|=1$

$$
\left|1+A_{k, \mu} w(z)\right| \geq \frac{n\left(|\alpha|-A_{k, \mu}\right)|p(z)|}{\left|D_{\alpha} p(z)\right|} .
$$

This implies for $0 \leq \theta<2 \pi$,

$$
n\left(|\alpha|-A_{k, \mu}\right)\left|p\left(e^{i \theta}\right)\right| \leq\left|1+A_{k, \mu} w\left(e^{i \theta}\right)\right|\left|D_{\alpha} p\left(e^{i \theta}\right)\right| .
$$

This gives for each $r>0$

$$
n^{r}\left(|\alpha|-A_{k, \mu}\right)^{r}\left|p\left(e^{i \theta}\right)\right|^{r} \leq\left|1+A_{k, \mu} w\left(e^{i \theta}\right)\right|^{r}\left|D_{\alpha} p\left(e^{i \theta}\right)\right|^{r} .
$$

Integrating both sides of the above inequality with respect to $\theta$, from 0 to $2 \pi$, we get

$$
\begin{aligned}
& n^{r}\left(|\alpha|-A_{k, \mu}\right)^{r} \int_{0}^{2 \pi}\left|p\left(e^{i \theta}\right)\right|^{r} d \theta \\
& \leq \int_{0}^{2 \pi}\left|1+A_{k, \mu} w\left(e^{i \theta}\right)\right|^{r}\left(\left|D_{\alpha} p\left(e^{i \theta}\right)\right|^{r}\right) d \theta .
\end{aligned}
$$

The above inequality on applying Holder's inequality for $p>1, q>1$ and $r>0$, yields

$$
\begin{aligned}
& n\left(|\alpha|-A_{k, \mu}\right)\left(\int_{0}^{2 \pi}\left|p\left(e^{i \theta}\right)\right|^{r} d \theta\right)^{\frac{1}{r}} \\
& \leq\left(\int_{0}^{2 \pi}\left|1+A_{k, \mu} w\left(e^{i \theta}\right)\right|^{p r} d \theta\right)^{\frac{1}{p r}}\left(\int_{0}^{2 \pi}\left|D_{\alpha} p\left(e^{i \theta}\right)\right|^{q r} d \theta\right)^{\frac{1}{q r}},
\end{aligned}
$$


Using inequality (3.6) with $r$ replaced by $p r$ in (3.8), we obtain for each $p>1, q>1$ and

$$
\begin{aligned}
r> & 0 \text { with } \frac{1}{p}+\frac{1}{q}=1, \\
& n\left(|\alpha|-A_{k, \mu}\right)\left(\int_{0}^{2 \pi}\left|p\left(e^{i \theta}\right)\right|^{r} d \theta\right)^{\frac{1}{r}} \\
& \leq\left(\int_{0}^{2 \pi}\left|1+A_{k, \mu} e^{i \theta}\right|^{p r} d \theta\right)^{\frac{1}{p r}}\left(\int_{0}^{2 \pi}\left|D_{\alpha} p\left(e^{i \theta}\right)\right|^{q r} d \theta\right)^{\frac{1}{q r}},
\end{aligned}
$$

which is the inequality (1.9) and this completes the proof of Theorem 1.

\section{Conclusion}

Bounds given by our theorem are better than the bounds obtained from inequality (1.8) and inequality (1.7) for polynomials having all zeros in $|z| \leq k, k \leq 1$.

\section{References:}

1. Zygmund, Proc. London Math. Soc. 34, 392 (1932).

https://doi.org/10.1112/plms/s2-34.1.392

2. V. V. Arestov, IZV. Akad. Nauk. SSSR. Ser. Math. 45, 3 (1981).

3. R. Lal, Ganita, 66, 57 (2016).

4. W. Rudin, Real and Complex Analysis (Tata McGraw-Hill Publishing Company, India, 1977).

5. S. Bernstein, Lecons Sur Les Proprietes extrremales et la meillure approximation des functions analytiquesd'une functions reele, Paris, 1926.

6. N. G. de-Bruijn, Inequalties Concerning Polyin the Complex Domain, Nederl. Akad. Wetench. Proc. Ser. A 50, 1265 (1947); Indag. Math. 9, 591 (1947).

7. Q. I. Rahman, Trans. Am. Math. Soc. 115, 337 (1992). https://doi.org/10.1090/S0002-9939-1992-1113648-1

8. A. Aziz and N. A. Rather, Math. Inequal. Appl. 1, 231 (1998). https://doi.org/10.7153/mia-01-21

9. N. K. Govil and G. N. Nyuydinkong, and B. Tameru, J. Math. Anal. Appl. 254, 618 (2001). https://doi.org/10.1006/jmaa.2000.7267

10. K. K. Dewan and C. M. Upadhye, J. Inequal. Pur Appl. Math. 9, Article ID 119 (2008).

11. A. Aziz and W. M. Shah, Ind. J. Pure Appl. Math. 28, 1413 (1997).

12. A. Aziz and N. A. Rather, J. Math. Anal. Appl. 289, 14 (2004). https://doi.org/10.1016/S0022-247X(03)00530-4

13. E. Hille, Analytic Function Theory (Ginn and Company, New York, 1962) II. 